\title{
White dwarf atmosphere models with Ly-alpha opacity in the analysis of the white dwarf cooling sequence of NGC 6397
}

\author{
P. M. Kowalski
}

\begin{abstract}
Lehrstuhl für Theoretische Chemie, Ruhr-Universität, 44780 Bochum, Germany
\end{abstract}
e-mail: piotr.kowalski@theochem.ruhr-uni-bochum.de

Received 31 May 2007 / Accepted 27 June 2007

\section{ABSTRACT}

\begin{abstract}
Aims. We discuss the importance of pure hydrogen white dwarf atmosphere models with Ly- $\alpha$ far red wing opacity in the analysis of the white dwarf cooling sequence of the globular cluster NGC 6397.

Methods. Our recently improved atmosphere models account for the previously missing opacity from the Ly- $\alpha$ hydrogen line broadened by collisions of the absorbing hydrogen atoms with molecular and atomic hydrogen. These models are the first that well reproduce the UV colors and spectral energy distributions of cool white dwarfs with $T_{\text {eff }}<6000 \mathrm{~K}$ observed in the Galactic Disk.

Results. Fitting the observed $F 814 W$ magnitude and $F 606 W-F 814 W$ color we obtained a value for the true distance modulus, $\mu=12.00 \pm 0.02$, that is in agreement with recent analyses. We show that the stars at the end of the cooling sequence appear to be $\sim 160 \mathrm{~K}$ cooler when models that account for Ly- $\alpha$ opacity are used. This indicates that the age of NGC 6397 derived from the white dwarf cooling sequence using atmosphere models that do not include the correct Ly- $\alpha$ opacity is underestimated by $\sim 0.5 \mathrm{Gyr}$. Conclusions. Our analysis shows that it is essential to use white dwarf atmosphere models with Ly- $\alpha$ opacity for precise dating of old stellar populations from white dwarf cooling sequences.
\end{abstract}

Key words. stars: white dwarfs - stars: atmospheres - Galaxy: globular clusters: individual: NGC 6397

\section{Introduction}

Globular clusters are the oldest known stellar systems in our Galaxy. They contain a large population of very cool and very old white dwarfs, remnants of stars that formed and died through the history of a cluster. Such populations have been investigated in the two nearest globular clusters: M4 (Hansen et al. 2002; Richter et al. 2004; Hansen et al. 2004) and NGC 6397 (Hansen et al. 2007; Richer et al. 2006). Recent data on NGC 6397 show a clear white dwarf cooling sequence and a low luminosity cut off related to the finite age of the cluster. On the basis of this sequence, Hansen et al. (2007) derived the true distance modulus $\mu=12.02 \pm 0.06$, the reddening toward NGC 6397 $E(F 606 W-F 814)=0.20 \pm 0.03$, and its age $T_{\mathrm{c}}=11.47 \pm$ 0.47 Gyr. The detailed and extended analysis presented in that publication gives the first precise and independent dating of a globular cluster based on its white dwarf cooling sequence.

Reliable atmosphere models need to be used in such analyses (Fontaine et al. 2001; Hansen 1999). Models currently in use (e.g. Bergeron et al. 2001; Saumon \& Jacobson 1999; Hansen 1998; Bergeron et al. 1995), although valuable in the analysis of cool white dwarfs (e.g. Bergeron et al. 2001), have a well known shortcoming. They cannot correctly reproduce the UV flux $(\lambda<5000 \AA)$ of white dwarfs with $T_{\text {eff }}<6000 \mathrm{~K}$ (Bergeron 2001; Bergeron et al. 2001, 1997). This problem has been solved recently by introducing the far red wing profile of hydrogen Ly- $\alpha$ into the modeling. The line profile is formed by collisions between absorbing hydrogen atoms and the atomic and molecular hydrogen, and when introduced into the modeling it allows for an accurate reproduction of the spectral energy distribution of cool white dwarfs at all wavelengths (Kowalski \& Saumon 2006). The synthetic spectra resulting from our improved pure hydrogen atmosphere models deviate significantly from the previous ones for wavelengths shorter than $1 \mu \mathrm{m}$ (Fig. 1). This indicates that the Ly- $\alpha$ opacity also affects the flux in filters $F 606 \mathrm{~W}$ and $F 814 W$ used to obtain the photometric data on the white dwarf cooling sequence of NGC 6397.

In this paper we discuss the importance of our new white dwarf atmosphere models with Ly- $\alpha$ opacity in the analysis of white dwarf cooling sequences. Fitting the theoretical to observed white dwarf cooling sequence of NGC 6397 we derived the true distance modulus to the cluster. We show that its value is consistent with that obtained with old models. We also predict that the coolest white dwarfs observed in NGC 6397 are cooler, when the atmosphere models with Ly- $\alpha$ opacity are used in the analysis. This implies that the cluster age derived from the white dwarf cooling sequence by Hansen et al. (2007) is probably too low. Comparing the temperature of the white dwarfs at the cut off of the white dwarf cooling sequence in NGC 6397 and the galactic disk we show that the local population of disk white dwarfs is much younger than that observed in the globular cluster.

\section{Cool white dwarf atmosphere models}

Hansen et al. (2007) and Richer et al. (2006) in their analysis of the white dwarf cooling sequence of NGC 6397 used the atmosphere models of Bergeron et al. (2001). However, there is a well known shortcoming of these models, as they overestimate the flux at short wavelengths $(\lambda<5000 \AA$, see Bergeron et al. 1997; Bergeron 2001). We have corrected this deficiency by introducing the opacity of the far red wing of the Ly- $\alpha$ broadened by collisions of the absorbing hydrogen atoms with atomic and molecular hydrogen (Kowalski \& Saumon 2006). Our new models allow for fits to the entire spectral energy distribution of white 

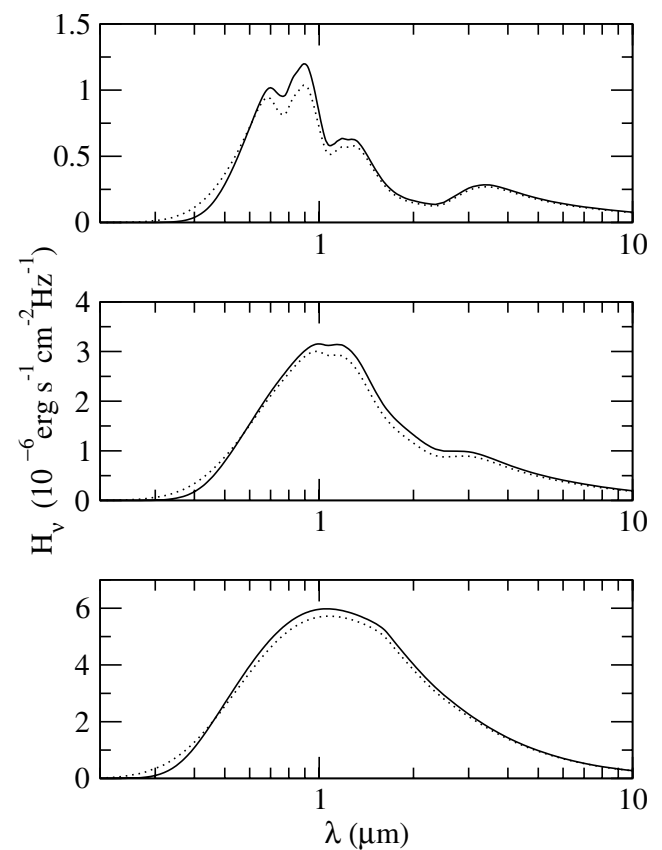

Fig. 1. Synthetic spectra of pure hydrogen atmosphere models with $T_{\text {eff }}=3000 \mathrm{~K}, 4000 \mathrm{~K}$, and $5000 \mathrm{~K}$ (from top to bottom), and fixed gravity $\log g=8$ (cgs) calculated with (solid) and without (dotted) Ly- $\alpha$ far red wing opacity. The dotted lines mimic the models of Bergeron et al. (1995) and Saumon \& Jacobson (1999) used by Hansen et al. (2007) and Richer et al. (2006) in their analysis of the white dwarf cooling sequence of NGC 6397.

dwarfs observed in our Galaxy, including the fit to the near-UV HST spectrum of the white dwarf star BMP 4729 (Kowalski \& Saumon 2006; Koester \& Wolff 2000), and the reproduction of the observed white dwarf color sequences below $T_{\text {eff }} \sim 6000 \mathrm{~K}$ (Kowalski \& Saumon 2006).

In Fig. 1 we present the impact of the Ly- $\alpha$ opacity on the synthetic spectra of cool white dwarfs. The flux suppressed by the Ly- $\alpha$ opacity at short wavelengths is redistributed towards longer wavelengths (well beyond $\lambda \sim 1 \mu \mathrm{m}$ ). This causes a non negligible increase of the flux at visible and near infrared wavelengths. As a result, the synthetic photometry in the $F 606 \mathrm{~W}$ and $F 814 W$ filters is also affected. This is shown in Fig. 2 where we present the color-magnitude diagram for a cooling sequence of a white dwarf of mass $0.5 M_{\odot}$ computed using models with and without the Ly- $\alpha$ opacity. Both curves deviate significantly from each other for $T_{\text {eff }}<5000 \mathrm{~K}$. As Ly- $\alpha$ opacity is stronger at short wavelengths, the effect is stronger in the $F 606 \mathrm{~W}$ filter (larger horizontal shift in Fig. 2). The new models predict a turn towards the blue, caused by CIA opacity from molecular hydrogen (Saumon \& Jacobson 1999; Hansen 1998; Bergeron et al. $1995)$, at lower $T_{\text {eff. }}$ This should have an impact on the astronomical parameters of NGC 6397 derived from the analysis of its white dwarf cooling sequence.

\section{Analysis of the data}

\subsection{The fit to the white dwarf cooling sequence}

To investigate the importance of pure hydrogen white dwarf atmosphere models with Ly- $\alpha$ opacity in the analysis of the white dwarf cooling sequence of NGC 6397 we performed a least square 2D fit in $F 814 W$ and $F 606 W-F 814 W$ to the photometric data of Hansen et al. (2007); Richer et al. (2006); their Fig. 6

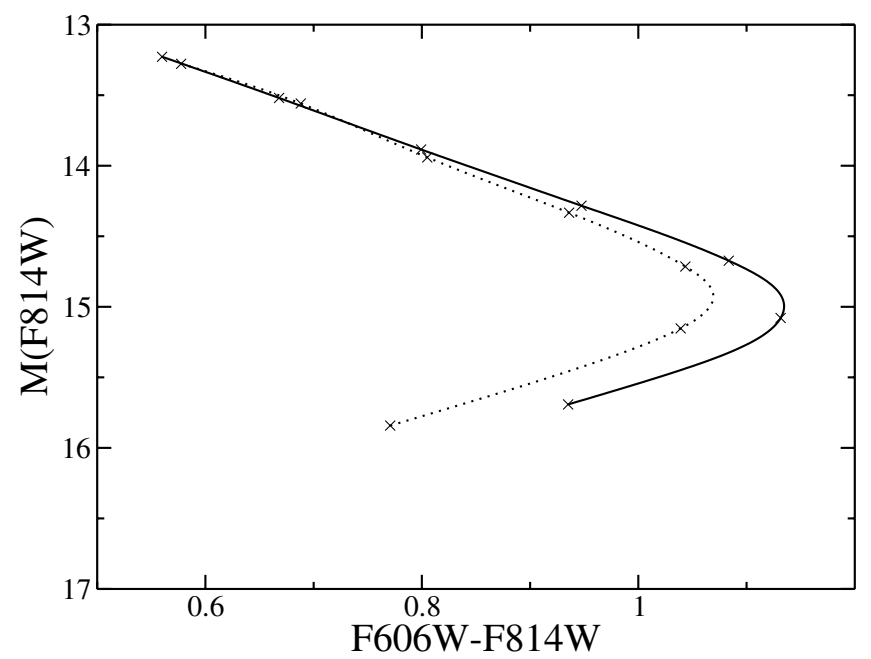

Fig. 2. Color-magnitude diagram of the white dwarf cooling sequence. The mass of the star is $0.5 M_{\odot}$, and the effective temperature runs from $6000 \mathrm{~K}$ (top) to $3000 \mathrm{~K}$ (bottom). Crosses indicate the $T_{\text {eff }}$ with $500 \mathrm{~K}$ decrements along the curves. Predictions of both models with (solid) and without (dotted) Ly- $\alpha$ opacity are shown. On the vertical axis the absolute magnitude is indicated.

and 4 respectively. The fitting parameters were the reddening and the true distance modulus. The fit was performed using two grids of atmosphere models: those with Ly- $\alpha$ opacity and those without. The second set mimics the models used in the analysis of the cluster white dwarf cooling sequence conducted by Hansen et al. (2007) and Richer et al. (2006). Following those authors, in our derivation of the theoretical cooling sequences we assumed the mass of the white dwarf to be $0.5 M_{\odot}$. Our fits to the data are given in Fig. 3. We obtained perfect fits with both theoretical cooling sequences, as is also the case in Hansen et al. (2007) and Richer et al. (2006). In addition to that work we show, for the first time, the $T_{\text {eff }}$ of cool white dwarfs in the globular cluster. Because of the difference in localization of the turn off visible in both theoretical sequences (Fig. 2), both fits give different estimations of $T_{\text {eff }}$ for the cool end of the observed sequence localized at $F 814 W \sim 27.6$ (Hansen et al. 2007). The effective temperature estimated on the basis of atmosphere models that account for Ly- $\alpha$ opacity is significantly smaller, indicating that the stars at the cut off of the observed white dwarf cooling sequence are cooler and older. We will return to this problem in the next section.

\subsection{Reddening and the true distance modulus}

As indicated in Fig. 2 there is a significant variation in the modeled maximum value of the $F 606 W-F 814 W$ color for a white dwarf of a given mass. In the present case, both model curves deviates by $\sim 0.1 \mathrm{mag}$, which has a direct impact on the derived value for the reddening towards NGC 6397. With models that do not account for the Ly- $\alpha$ opacity we obtained $E(F 606 W-F 814 W)=0.18 \pm 0.2$ with a good agreement with the value 0.16 obtained by Hansen et al. (2007). Models with the Ly- $\alpha$ give a lower value of $0.12 \pm 0.02$. This result, when converted from the ACS photometric system to the ground-based colors using the prescription of Sirianni et al. (2005) gives $E(B-V)=0.13 \pm 0.03$. This value is lower than $E(B-$ $V)=0.18$ obtained by Gratton et al. (2003) using a fit to the Main Sequence stars. However, the reddening obtained by 


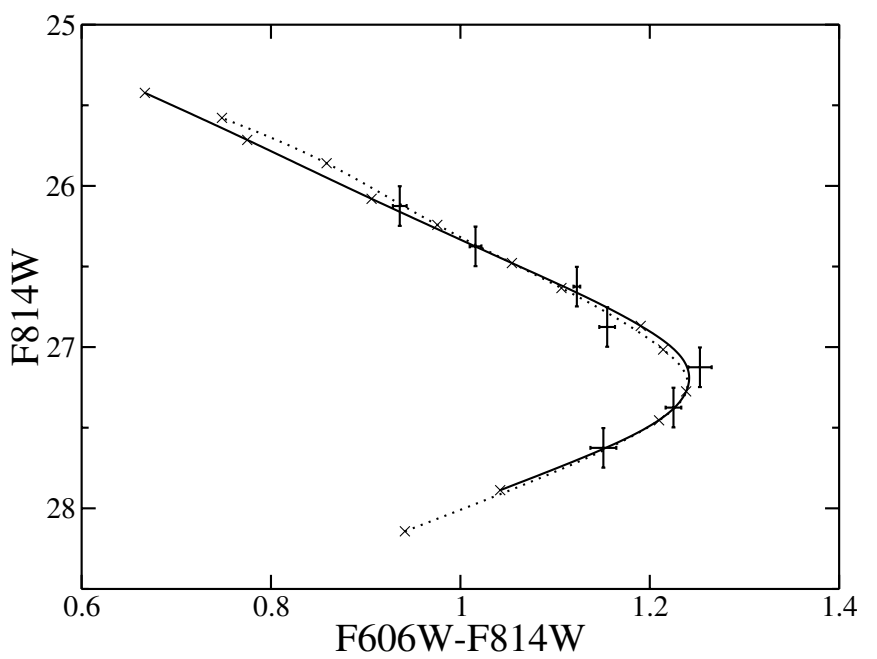

Fig. 3. Color-magnitude diagram of the white dwarf cooling sequence observed in NGC 6397. The lines represent the fits to data using modeled cooling sequences for a white dwarf of mass $0.5 M_{\odot}$. The effective temperature runs from $6000 \mathrm{~K}$ (top) to $3000 \mathrm{~K}$ (bottom). Small crosses indicate the $T_{\text {eff }}$ with $500 \mathrm{~K}$ decrements along the curves. Models with (solid) and without (dotted) Ly- $\alpha$ opacity are shown.

Table 1. Effective temperature and ages of white dwarfs at the end of the cooling sequence of NGC 6397.

\begin{tabular}{|c|c|c|c|c|}
\hline mass $\left(M_{\odot}\right)$ & $T_{\text {eff }}(\mathrm{K})$ & age (Gyr) & $T_{\text {eff }}(\mathrm{K})$ & age (Gyr) \\
\hline & \multicolumn{2}{|c|}{ models with Ly- $\alpha$} & \multicolumn{2}{|c|}{ models without Ly- $\alpha$} \\
\hline 0.5 & $3180 \pm 80$ & $9.8 \pm 0.4$ & $3340 \pm 80$ & $9.2 \pm 0.3$ \\
\hline 0.6 & $3210 \pm 80$ & $11.1 \pm 0.3$ & $3380 \pm 90$ & $10.6 \pm 0.3$ \\
\hline
\end{tabular}

Table 2. Effective temperature of the white dwarfs at the end of the cooling sequence observed in the Galactic Disk.

\begin{tabular}{ccc}
\hline \hline color & value & $T_{\text {eff }}(\mathrm{K})$ \\
\hline$B-V$ & $1.4 \pm 0.1$ & $3750 \pm 250$ \\
$V-I$ & $1.4 \pm 0.1$ & $3800 \pm 300$ \\
$u-g$ & $2.6 \pm 0.1$ & $3750 \pm 150$ \\
$g-z$ & $1.9 \pm 0.1$ & $3850 \pm 150$ \\
\hline
\end{tabular}

fitting the $M=0.5 M_{\odot}$ theoretical cooling sequence to the photometric data is expected to be underestimated. This is because of the variation in the average mass of a white dwarf at the end of the cooling sequence. This is discussed in detail by Hansen et al. (2007). The final reddening derived in that paper is $E(F 606 W-F 814 W)=0.20 \pm 0.3$. This is $\sim 0.04$ dex larger than the value they obtained using the $M=0.5 M_{\odot}$ theoretical cooling sequence. Applying such a shift to our result, we obtain $E(F 606 W-F 814 W)=0.16 \pm 0.02$ and $E(B-V)=0.17 \pm 0.03$ respectively. This value is in good agreement with the result of Gratton et al. (2003).

For the true distance modulus, in both fits we obtained a consistent value of $12.00 \pm 0.02$, which agrees with the value obtained by Hansen et al. (2007), $\mu=12.02 \pm 0.06$. Our estimate of the true distance modulus was obtained assuming a single white dwarf mass of $M=0.5 M_{\odot}$ and the relation between reddening in both filters $A_{814}=0.65 A_{606}$ (Sirianni et al. 2005). When we use the $M=0.6 M_{\odot}$ mass star instead, the resulting distance modulus is lower, $11.85 \pm 0.02$.

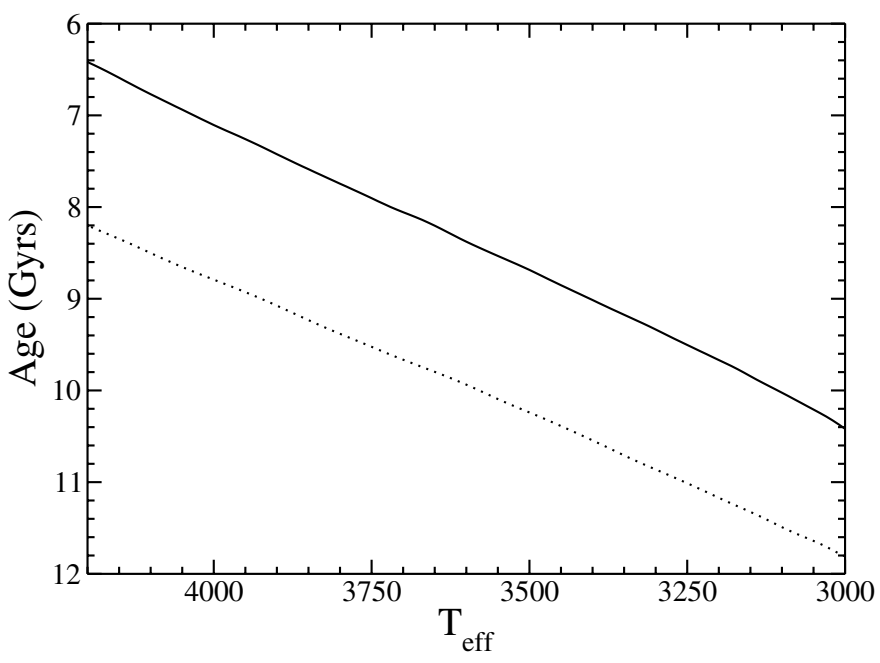

Fig. 4. White dwarf cooling times vs. $T_{\text {eff }}$ for a white dwarf of mass $0.5 M_{\odot}$ (solid line) and $0.6 M_{\odot}$ (dotted line). Models of Richer et al. (2000).

\section{4. $T_{\text {eff }}$ at the edge of the white dwarf cooling sequence and the age of NGC 6397}

The proper derivation of the age of NGC 6397 from its white dwarf cooling sequence is a difficult task and is beyond the scope of this paper. However, as use of the new atmosphere models shifts the effective temperature of the coolest white dwarfs by a few hundred degrees (Fig. 2), it is important to show how large a bias this effect would produce on the derived age of NGC 6397. To check this we conducted a simple exercise, similar to that of Hansen et al. (2007), Appendix A. Following the authors we assumed that the observed cut off of the white dwarf cooling sequence takes place at $F 814 W=27.6 \pm 0.1$. Then assuming the white dwarf masses to be $0.5 M_{\odot}$ and $0.6 M_{\odot}$ respectively we derived the $T_{\text {eff }}$ and, using the same white dwarf cooling models of Richer et al. (2000) as Hansen et al. (2007), we derive the cooling times of the white dwarfs at the cooling sequence cut off. The results are summarized in Table 1 . The $T_{\text {eff }}$ and the ages derived using atmosphere models with and without Ly- $\alpha$ opacity differ by $\sim 160 \mathrm{~K}$ and $\sim 0.5 \mathrm{Gyr}$ respectively, and these differences are insensitive to the assumed white dwarf mass. This suggests that the age of the globular cluster derived in Hansen et al. (2007) can be $\sim 0.5$ Gyr too low because of the white dwarf atmosphere models that do not account for Ly- $\alpha$ red wing opacity.

We also conducted the same simple analysis for the white dwarf cooling sequence observed in the Milky Way obtained from three samples of white dwarfs (Bergeron et al. 2001; Kilic et al. 2006; Harris et al. 2006). To do this we used published color sequences of Kowalski \& Saumon (2006). The obtained value of colors and $T_{\text {eff }}$ at the cut off are given in Table 2. Our analysis shows that the coolest white dwarfs observed in the Galactic Disk have $T_{\text {eff }} \sim 3800 \mathrm{~K}$. This indicates that the coolest stars in the local population of white dwarfs are warmer by $\sim 600 \mathrm{~K}$ than the coolest white dwarfs observed in NGC 6397. According to white dwarf cooling models of Richer et al. (2000) such a difference in effective temperature results in $\sim 2 \mathrm{Gyr}$ difference between the age of the white dwarfs in NGC 6397 and the local white dwarf population (Fig. 4). This is consistent with the result of Fontaine et al. (2001), who, based on the 
analysis of the white dwarf luminosity functions, concluded that the Galactic Halo is 2-3 Gyr older than the Galactic Disk.

\section{Conclusions}

Observation of the white dwarf cooling sequences in the globular clusters opens the possibility to date them more precisely. However, there are many factors that affect the models applied to white dwarf cosmochronology. Here we have discussed the impact of white dwarf atmosphere models on the derivation of the basic astronomical properties of NGC 6397 from the white dwarf cooling sequence itself. Our most recent set of pure hydrogen models, that corrects for the flux discrepancy at short wavelengths, results in lower reddening toward the cluster, and older derived ages. By deriving the effective temperature of white dwarfs at the end of the white dwarf cooling sequences of NGC 6397 and the Galactic Disk we have also shown that the coolest white dwarfs observed in the globular cluster are $\sim 600 \mathrm{~K}$ cooler, and therefore $\sim 2$ Gyr older. More sophisticated analysis that includes the construction of the white dwarf luminosity function is necessary for correct derivation of the cluster age from the white dwarf cooling sequence; however, our analysis shows that atmosphere models with Ly- $\alpha$ opacity are necessary to reliably estimate the ages of old stellar populations from the white dwarf cooling sequences ${ }^{1}$.
Acknowledgements. I thank Didier Saumon for useful comments on this manuscript and the theoretical chemistry group at Ruhr University in Bochum for hospitality and support.

\section{References}

Bergeron, P. 2001, ApJ, 558, 369

Bergeron, P., Saumon, D., \& Wesemael, F. 1995, ApJ, 443, 764

Bergeron, P., Ruiz, M. T., \& Leggett, S. K. 1997, ApJ, 108, 339

Bergeron, P., Leggett, S. K., \& Ruiz, M. T. 2001, ApJS, 133, 433

Fontaine, G., Brassard, P. \& Bergeron, P. 2001, PASP, 113, 409

Gratton, R. G., Bragaglia, A., Carretta, E., et al. 2003, A\&A, 408, 529

Hansen, B. 1999, ApJ, 520, 680

Hansen, B. M. S. 1998, Nature, 394, 860

Hansen, B. M. S., et al. 2002, ApJ, 574, L155

Hansen, B., Hansen, B. M. S., Richer, H. B., Fahlman, G. G., et al. 2004, ApJS, 155,551

Hansen, B., Hansen, B. M. S., Anderson, J., Brewer, J., et al. 2007, [arXiv: astro-ph/0701738]

Harris, H. C., Harris, H. C., Munn, J. A., Kilic, M., et al. 2006, ApJ, 131, 571

Kilic, M., von Hippel, T., Leggett, S. K., Winget, D. E. 2006, ApJ, 646, 474

Koester, D. \& Wolff, B. 2000, A\&A, 357, 587

Kowalski, P. M., \& Saumon, D. 2006, ApJ, 651, L137

Richer, H. B., Hansen, B., Limongi, M., et al. 2000, ApJ, 529, 318

Richer, H. B., Brewer, J., Fahlman, G. G., et al. 2004, AJ, 127, 2904

Richer, H. B., Anderson, J., Brewer, J., et al. 2006, Science, 313, 936

Saumon, D., \& Jacobson 1999, ApJ, 511, L107

Sirianni, M., Sirianni, M., Jee, M. J., Beni'tez, N., et al. 2005, PASP, 117, 1049

${ }^{1}$ Colors available upon request. 